\title{
MedienPädagogik
}

Zeitschrift für Theorie und Praxis der Medienbildung

Themenheft Nr. 36: Teilhabe in einer durch digitale Medien geprägten Welt Perspektiven des wissenschaftlichen Nachwuchses

Herausgegeben von Tim Riplinger, Jan Hellriegel und Ricarda Bolten

\section{E-Teaching Kompetenz von Fachhochschullehrenden}

\section{Ergebnisse einer formativen Evaluation an der Hochschule für Wirtschaft und Gesellschaft Ludwigshafen}

Imke Buß und Anne Keller

\begin{abstract}
Zusammenfassung
Virtuelles Lehren und Lernen wird seit Jahren politisch gefordert und gefördert. Dennoch ist der Alltag an Hochschulen immer noch stark geprägt von Präsenzlehre und virtuelle Lehre stellt oft nur eine Ergänzung zu dieser dar. Die Analysen dieses Artikels beziehen sich auf eine Befragung von Hochschullehrenden an der Hochschule für Wirtschaft und Gesellschaft Ludwigshafen, die im Jahr 2017 durchgeführt wurde ( $N=67)$. Im Fokus der Untersuchung steht die Frage, wie die digitalen Kompetenzen von Hochschullehrenden in Bezug auf virtuelle Lehre einzuschätzen sind und wie diese Kompetenzen mit eigenen Lehrerfahrungen sowie der Offenheit oder Skepsis gegenüber virtueller Lehre zusammenhängen. Es zeigt sich, dass Professorinnen und Professoren im Vergleich zu Lehrbeauftragten deutlich häufiger virtuell lehren und ihre E-Teaching Kompetenzen generell höher einschätzen. Ausserdem sind diejenigen E-Teaching Kompetenzen besonders gering ausgeprägt, die für einen systematischen Ersatz von Präsenzlehre durch virtuelle Lehre erforderlich wären. Schliesslich wird deutlich, dass die intrinsische Motivation mit der Nutzungshäufigkeit korreliert, nicht jedoch die extrinsische Motivation.
\end{abstract}

\section{E-Teaching Competencies of University Lecturers}

\begin{abstract}
E-Teaching and E-Learning have been both demanded and promoted by the government for years. Nevertheless, universities still rely largely on classroom teaching, and digital teaching serves only as a supplementary strategy. The findings of this article are based on a survey of professors and lecturers at the Ludwigshafen University of Business and Society conducted in 2017 ( $N=67)$. Central questions are: How can the digital teaching skills of university lecturers be judged and how do their digital competencies relate to their own educational experience as well as to their stance on digital teaching? The findings show a large discrepancy between full-time (assistant) professors and assistant lecturers in terms of implementation as well as in terms of competence. Furthermore,
\end{abstract}


e-teaching competencies that would be required for digital teaching to replace classroom teaching are on a low level. Lastly, intrinsic motivation correlates with the frequent use of e-teaching, while the extrinsic motivation is irrelevant.

\section{Einleitung}

Virtuelles Lehren und Lernen wird von der Politik seit Jahren als Standard proklamiert und schon seit 1998 durch zahlreiche Projektförderungen von Bund und Ländern unterstützt (Kleimann und Wannemacher 2004; Busse 2017). Hierdurch sind die Hochschulen aufgefordert, ihre überwiegend auf Präsenzlehre ausgerichteten Studiengänge durch E- oder Blended-Learning Konzepte weiterzuentwickeln (Arnold et al. 2018). Dies soll insbesondere auch einer Flexibilisierung des Studiums dienen, individuellen Lerngeschwindigkeiten verschiedener Studierendengruppen Rechnung tragen und somit die Hochschulen insgesamt attraktiver machen (Kultusministerkonferenz 2016). In der Realität zeigt sich allerdings, dass bisher nur ein Bruchteil der Lehrenden solche Konzepte umsetzt und Lernmanagementsysteme primär zur Ergänzung der Präsenzlehre genutzt werden (Carstensen 2009). Der «Monitor Digitale Bildung» der Bertelsmann Stiftung zeigt, dass zwar 92\% der Lehrenden digitale Präsentationstools (z.B. PowerPoint) nutzen, allerdings nur die Hälfte der Lehrenden Lernmanagementsysteme einsetzen (Schmid et al. 2017, 16). Die Gründe hierfür sind vielfältig und liegen einerseits in fehlendem Interesse, einer skeptischen Haltung bezüglich der Digitalisierung von Lehre oder fehlenden Kompetenzen (Hugger und Vollbrecht 2001). Andererseits befinden sich viele Hochschulen noch in einer frühen Phase der Digitalisierung (Phase der Pionierprojekte oder Phase der kooperativen Ansätze) und haben daher ihre Lehre noch nicht systematisch digitalisiert (Kerres und Getto 2017). Eine wichtige Voraussetzung für die erfolgreiche Gestaltung von virtuellen Lehr-Lernszenarien setzt an den Kompetenzen von Lehrenden und Studierenden an. Kreidl (2011) zeigt, dass die didaktische Konzeption und das Feedback der Lehrenden die studentische Akzeptanz der virtuellen Lehr-Lernsettings massgeblich beeinflussen. Die Konzeption von virtuellen Lehr-Lernszenarien kann allerdings nur dann eine gute Qualität aufweisen, wenn die Lehrenden hohe E-Teaching Kompetenzen aufweisen. Zahlreiche Autorinnen und Autoren beschäftigen sich mit der Frage, wie mediendidaktische, E-Teaching oder digitale Kompetenzen in der Lehrerbildung (Tulodziecki 2012) und der Hochschuldidaktik (Horvath 2009; Eichhorn, Müller und Tillmann 2017) zu definieren sind. Wenn die Lehrenden nicht kompetent mit virtueller Lehre umgehen können, kann dies zu Unsicherheiten und Widerständen gegen die Einführung derselben führen (Hugger 2004). Es lag bisher nur wenig empirische Forschung zu der Einschätzung der digitalen (Lehr-) Kompetenz von Hochschullehrenden und den Zusammenhängen mit anderen Faktoren wie Erfahrungen mit virtueller Lehre, Lehreinstellungen oder Motivation vor. Erfreulicherweise hat sich 
dies in den letzten zwei Jahren verbessert und es liegen zunehmend Forschungen vor, die sich auch im Kontext der Digitalisierung an Hochschulen mit diesem Thema befassen (Eichhorn und Tillmann 2018). Viele wissenschaftlichen Studien beziehen sich hingegen auf die Erfassung der Medienkompetenz von Jugendlichen (z.B. Medienprofis-Test) und Erwachsenen (z.B. Technologische und informationsbezogener Literacy-Test im Rahmen des Nationalen Bildungspanels) (Hermida, Hielscher und Petko 2017).

Im Rahmen einer formativen Evaluation der E-Learning- und hochschuldidaktischen Angebote der Hochschule für Wirtschaft und Gesellschaft Ludwigshafen und im Projekt «Offenes Studienmodell Ludwigshafen» konnten interessante Erkenntnisse gewonnen werden, die einen Beitrag zur Bearbeitung dieser Forschungslücke bieten. Diese Arbeit stellt die Frage, wie die E-Teaching Kompetenzen von Hochschullehrenden in Bezug auf die Durchführung von E-Learning einzuschätzen sind und wie E-Teaching Kompetenzen mit eigenen Lehrerfahrungen sowie der Offenheit oder Skepsis gegenüber virtueller Lehre zusammenhängen. Diese Fragestellung wird anhand der im Jahr 2017 erhobenen Daten analysiert.

\section{Medienkompetenz, E-Teaching Kompetenz oder digitale Kompetenz}

Hochschullehrende haben vielfältige Aufgaben in Bezug auf die Medienkompetenz von Studierenden. Sie sollen zum einen deren generelle Medienkompetenz fördern und fachspezifisch eine kompetente Mediennutzung anleiten (Kultusministerkonferenz 2016). Hierfür benötigen die Hochschullehrenden selbst entsprechende Kompetenzen, die sich auch auf die aktuellen Anforderungen und Diskurse im Fach beziehen. Bezogen auf die Mediennutzung kann dies z.B. die Kenntnisse über Datenanalyse mit Big Data in der Statistik, Psychologie oder Informatik beinhalten; bezogen auf das sichere Agieren in digitalen Umgebungen die Anwendung von Datenschutz- und Urheberrechtsvorschriften auf das zukünftige berufliche Handeln. Die KMK sieht es als besondere Chance, den Umgang mit und die Anwendung von digitalen Medien und Werkzeugen «über die Studierenden mittels digitaler Technologie intensiv und interaktiv in Lehr-Lern-Prozessen [zu fördern]» (Kultusministerkonferenz 2016, 46). Um die Lehr-Lernprozesse mittels digitaler Technologie im Sinne des E- oder Blended-Learnings zu gestalten, benötigen die Lehrenden mediendidaktische oder ETeaching-Kompetenzen.

Für das Lehren und Lernen mit digitalen Medien und in Lernmanagementsystemen sind über die genannten Kompetenzen hinaus weitere Kenntnisse und Fähigkeiten notwendig, welche die Konzeption und Umsetzung von virtueller Lehre ermöglichen. E-Learning beschreibt dabei »ein vielgestaltiges gegenständliches und organisatorisches Arrangement von elektronischen bzw. digitalen Medien zum Lernen» (Arnold et al. 2018, 22). In Hochschulen, in denen bisher Präsenzlehre vorherrscht, 
liegt der Fokus allerdings auf einer Kombination von E-Learning und Präsenzveranstaltungen, dem so genannten Blended-Learning. Dies «ist ein integriertes Lernkonzept, das die heute verfügbaren klassischen Lehrmethoden und -medien in einem sinnvollen Lernarrangement optimal nutzt. Es ermöglicht Lernen, Kommunizieren, Informieren und Wissensmanagement, losgelöst von Ort und Zeit in Kombination mit Erfahrungsaustausch, Rollenspiel und persönlichen Begegnungen im klassischen Präsenztraining» (Sauter, Annette, Sauter, Werner und Bender 2003, 68). Um solche Blended-Learning Szenarien zu entwickeln, sollten die Hochschullehrenden reflektieren können, welche Stärken und Schwächen Präsenz- und Onlinelehre haben.

Bis dato sind viele Begrifflichkeiten noch immer diffus und nicht einheitlich definiert, wie Dengel (2018) beispielsweise für den Begriff der Digitalen Bildung erläutert. Dies ist ebenso für die von den Lehrenden für das E-Learning erforderlichen Kompetenzen der Fall. Diese werden häufig als E-Teaching, mediendidaktische- oder digitale Kompetenzen bezeichnet. Die mediendidaktischen Handlungskompetenzen stellen eine Weiterentwicklung der Medienkompetenzen für den Anwendungsbereich der Lehre dar, beziehen sich aber nicht explizit auf E-Learning in der Hochschullehre. Es existieren international wie national mehrere Definitionen von Digitalen oder E-Teaching Kompetenzen, die Lehrende im Schul- oder Hochschulsystem benötigen. Die Definitionen unterscheiden sich in mehreren Aspekten. So fokussiert der UNESCO ICT Competency Framework for Teachers auf digitale Lernumgebungen, die Inklusion ermöglichen und OER, Virtual und Augmented Reality Kompetenzen zur Datenanalyse (Big Data) sukzessive mit einbeziehen (United Nations Educational 2018). Der European Digital Competence Framework der Europäischen Kommission berücksichtigt auch die Förderung von Selbstlern- und digitalen Kompetenzen der Schülerinnen und Schüler (Redecker 2017). Beide Konzepte fokussieren stärker auf Schule oder Erwachsenenbildung, wohingegen Horvarth (2009) und Eichhorn, Müller und Tillmann (2017) Kompetenzen von Hochschullehrenden betrachten. Horvarth legt einen besonderen Wert auf die Interaktion mit Studierenden und benennt die Kommunikations- und Beratungskompetenz sowie die Kompetenz zur Steuerung von Teamprozessen als eigene Bereiche. Eichhorn, Müller und Tillmann (2017) greifen die Entwicklung des Konzeptes digitale Kompetenz (Ferrari 2012) aus den letzten Jahren auf und entwickeln ein entsprechendes Kompetenzraster für digitale Kompetenzen von Hochschullehrenden. Dieses Kompetenzraster umfasst acht Kompetenzfelder, wobei das Thema «digitale Wissenschaft» bzw. «Digitalisierung in der Wissenschaft und Forschung» im Vergleich mit den anderen Definitionen eine Besonderheit darstellt. Darüber hinaus differenzieren die Autoren die Kompetenzfelder nach drei Kompetenzstufen aus - eine ähnliche Abstufung ist auch im ICT Competency Framework zu beobachten. Die Kompetenzstufen orientieren sich an der Lernzieltaxonomie für kognitive Kompetenzen von Bloom und Engelhart (1976) und umfassen die Stufe 1 «Überblickswissen und Grundlagen», Stufe 2 «Praktische Anwendung» und 
Stufe 3 «Weitergabe und Anleitung Anderer». Die in den vier Modellen definierten Kompetenzfelder sind in Tabelle $1 \mathrm{im}$ Vergleich dargestellt. Die Zuordnungen berücksichtigen dabei die detaillierten Beschreibungen der Kompetenzen.

\begin{tabular}{|c|c|c|c|c|}
\hline Dimension & $\begin{array}{l}\text { Horvarth } \\
(2009)\end{array}$ & $\begin{array}{l}\text { UNESCO ICT } \\
\text { Competency } \\
\text { Framework } \\
(2018)\end{array}$ & $\begin{array}{l}\text { DigCompEdu } \\
(2017)\end{array}$ & $\begin{array}{l}\text { Eichhorn, Mül- } \\
\text { ler und Till- } \\
\text { mann (2017) }\end{array}$ \\
\hline $\begin{array}{l}\text { Allgemeine Päd- } \\
\text { agogische Kom- } \\
\text { petenz }\end{array}$ & $\begin{array}{l}\text { Didaktische } \\
\text { Kompetenz }\end{array}$ & Pedagogy & \multirow{3}{*}{$\begin{array}{l}\text { Teaching and } \\
\text { Learning }\end{array}$} & Digitale Lehre \\
\hline $\begin{array}{l}\text { Gestaltung von } \\
\text { Kollaboration } \\
\text { und Kommuni- } \\
\text { kation }\end{array}$ & $\begin{array}{l}\text { Kommunikati- } \\
\text { onskompetenz } \\
\text { Steuerung von } \\
\text { Teamprozessen }\end{array}$ & $\begin{array}{l}\text { Curriculum and } \\
\text { Assessment }\end{array}$ & & $\begin{array}{l}\text { Digital kommu- } \\
\text { nizieren und } \\
\text { kooperieren }\end{array}$ \\
\hline $\begin{array}{l}\text { Beratung von } \\
\text { Studierenden }\end{array}$ & $\begin{array}{l}\text { Beratungskom- } \\
\text { petenz }\end{array}$ & - & & - \\
\hline $\begin{array}{l}\text { Organisation mit } \\
\text { digitalen Medien }\end{array}$ & $\begin{array}{l}\text { Planungs- und } \\
\text { Organisations- } \\
\text { kompetenz }\end{array}$ & $\begin{array}{l}\text { Organization and } \\
\text { Administration }\end{array}$ & \multirow{3}{*}{$\begin{array}{l}\text { Professional } \\
\text { Engagement }\end{array}$} & - \\
\hline $\begin{array}{l}\text { Reflexion des } \\
\text { Lehrhandelns } \\
\text { und Weiterbil- } \\
\text { dung }\end{array}$ & $\begin{array}{l}\text { Didaktische } \\
\text { Kompetenz }\end{array}$ & $\begin{array}{l}\text { Teacher Professi- } \\
\text { onal Learning }\end{array}$ & & $\begin{array}{l}\text { Analysieren und } \\
\text { reflektieren }\end{array}$ \\
\hline \multirow{2}{*}{$\begin{array}{l}\text { Gestaltung von } \\
\text { Lehr-Lernszenari- } \\
\text { en unter Nutzung } \\
\text { von digitalen } \\
\text { Medien }\end{array}$} & $\begin{array}{l}\text { Mediendidakti- } \\
\text { sche } \\
\text { Kompetenz }\end{array}$ & $\begin{array}{l}\text { Understanding } \\
\text { ICT in } \\
\text { Education }\end{array}$ & & $\begin{array}{l}\text { Bedienen und } \\
\text { anwenden }\end{array}$ \\
\hline & - & $\begin{array}{l}\text { Curriculum and } \\
\text { Assessment }\end{array}$ & Assessment & - \\
\hline $\begin{array}{l}\text { Technische Kom- } \\
\text { petenz }\end{array}$ & $\begin{array}{l}\text { Medientechni- } \\
\text { sche } \\
\text { Kompetenz }\end{array}$ & $\begin{array}{l}\text { Application of } \\
\text { Digital Skills }\end{array}$ & $\begin{array}{l}\text { Digital Ressour- } \\
\text { ces }\end{array}$ & $\begin{array}{l}\text { Produzieren und } \\
\text { präsentieren }\end{array}$ \\
\hline $\begin{array}{l}\text { Kompetenter und } \\
\text { rechtssicherer } \\
\text { Umgang mit } \\
\text { Daten }\end{array}$ & $\begin{array}{l}\text { Rechtliche Kom- } \\
\text { petenz }\end{array}$ & - & - & $\begin{array}{l}\text { Digital informie- } \\
\text { ren und recher- } \\
\text { chieren } \\
\text { Digitale Identität } \\
\text { und Karrierepla- } \\
\text { nung } \\
\text { Digitale Wissen- } \\
\text { schaft }\end{array}$ \\
\hline $\begin{array}{l}\text { Lernprozesse der } \\
\text { Studierenden }\end{array}$ & - & - & $\begin{array}{l}\text { Empowering } \\
\text { Learners } \\
\text { Facilitating Lear- } \\
\text { ners Digital Com- } \\
\text { petence }\end{array}$ & - \\
\hline
\end{tabular}

Tab. 1.: Gegenüberstellung von vier Modellen für E-Teaching Kompetenzen. 
Die Befragung der Lehrenden, die diesem Artikel zugrunde liegt, orientiert sich an der Definition von Horvarth (2009), da diese auf den Hochschulbereich und das an der Hochschule vorherrschende Blended-Learning angepasst ist. Gleiches trifft auch auf die Kompetenzdefinition von Eichhorn, Müller und Tillmann (2017) zu. Diese Studie war allerdings noch nicht erschienen, als die dem Artikel zugrundeliegende Umfrage konzipiert wurde. Was versteht Horvarth nun unter E-Teaching Kompetenz? Um virtuelle Lehre einsetzen zu können, sollten Lehrende Lehrveranstaltungen mediendidaktisch konzipieren können, Lernplattformen selbst bedienen können, die Lern- und Kollaborationsprozesse der Studierenden anleiten und sie (ggf. auch online) beraten können. Schliesslich sollten Lehrende über die relevanten Kenntnisse im Urheberrecht und Datenschutz verfügen (Horvath 2009). Literatur zur Gestaltung von Qualifizierungsmassnahmen zur Durchführung von E-Learning Vorhaben kann hilfreiche Ergänzungen der vorliegenden Kompetenzdefinitionen liefern. So fokussiert Kohl (2004) neben den genannten Aspekten die Fähigkeit von Lehrenden, die Tauglichkeit von digitalen Medien für das jeweilige Lernsetting zu prüfen und lerngerechte Materialien zu erstellen. Diesem Beitrag liegt eine E-Teaching Definition zugrunde, welche die Ansätze von Horvarth (2009) und Kohl (2004) zusammenbringt (Tabelle 2).

\begin{tabular}{|l|l|}
\hline Kompetenzdimension & Kenntnisse, Fähigkeiten sowie Einstellungen \\
\hline Medientechnische Kompetenz & $\begin{array}{l}\text { Computer- und Internetnutzung, Nutzung von E-Learning } \\
\text { Tools (z.B. Lernplattformen), Beurteilung von Aufwand } \\
\text { und Tauglichkeit der Tools, Gestaltung von lerngerechten } \\
\text { Materialien. }\end{array}$ \\
\hline Mediendidaktische Kompetenz & $\begin{array}{l}\text { Mediendidaktische Planung und Konzeption von Lehr- } \\
\text { veranstaltungen sowie Test- und Prüfungsverfahren, (kriti- } \\
\text { sche) Reflexion des Medieneinsatzes in der Lehre }\end{array}$ \\
\hline Beratungskompetenz & Webgestützte Betreuung und Beratung der Studierenden \\
\hline Rechtliche Kompetenz & $\begin{array}{l}\text { Urheberrecht, Umgang mit Studierendendaten, Bereitstel- } \\
\text { lung von Material im Internet }\end{array}$ \\
\hline
\end{tabular}

Tab. 2.: Erhobene Dimensionen von E-Teaching Kompetenz nach Horvath (2009), durch die Autorinnen ergänzt durch kursiv geschriebene Kompetenzbereiche nach Kohl (2004).

\section{Hypothesen zu Umfang und Entwicklung von E-Teaching Kompetenz}

Das Ziel der Analysen war es herauszufinden, wie die E-Teaching Kompetenzen der Hochschullehrenden an der Hochschule für Wirtschaft und Gesellschaft Ludwigshafen derzeit ausgeprägt sind, in welchem Umfang die Angebote und Tools genutzt werden und welche Faktoren die Kompetenzen und die Nutzung beeinflussen. Das E-Learning Team der Hochschule hat in Beratungsgesprächen festgestellt, dass Präsenzlehre häufig durch virtuelle Elemente angereichert wird. Nur wenige Lehrende ersetzen Teile der Präsenzlehre durch virtuelle Lehre, was u.U. mit der erst seit Ende 2016 bestehenden Regelung zur Anrechnung von E-Learning auf das Lehrdeputat zusammenhängt (Lungershausen, Emunds und Buß 2016). Es ist daher davon 
auszugehen, dass diejenigen Kompetenzen besonders hoch ausgeprägt sind, welche Lehrende für die Anreicherung von Präsenzlehre benötigen (z.B. Nutzung von Lernplattformen, rechtliche Kompetenzen).

Hypothese 1: Es sind solche E-Teaching Kompetenzen besonders hoch ausgeprägt, die für die Anreicherung von Präsenzlehre nützlich sind.

Kompetenzen hängen eng mit Handlungen zusammen und entwickeln sich durch Lernen und Erfahrung weiter (Schöler 2016; Vonken 2015). Übertragen auf das ETeaching bedeutet dies, dass Lehrende virtuelle Lehre konzipieren, durchführen und dabei ihre E-Teaching Kompetenzen weiterentwickeln. Gleichzeitig ist lerntheoretisch belegt, dass je höher die Personen die eigenen Kompetenzen einschätzen es umso wahrscheinlicher wird, dass auch neue ggf. schwierige Aufgaben übernommen werden - was auch als Selbstwirksamkeitsdynamik beschrieben wird (Satow 2002). Die Hypothese 2 und 3 prüfen daher, ob die aktive Nutzung von virtueller Lehre mit höheren E-Teaching Kompetenzen zusammenhängt.

Hypothese 2: Eine aktive Nutzung von virtueller Lehre hängt mit höheren ETeaching Kompetenzen zusammen.

Hypothese 3: Hauptamtlich Lehrende verfügen über mehr Erfahrung im E-Teaching und schätzen daher ihre E-Teaching-Kompetenzen höher ein.

Die Hypothese vier befasst sich mit der Frage, wie Hochschullehrende zu einer häufigeren und kompetenten Durchführung von E-Learning motiviert werden können. Dies unterstützen Hochschulen u.a. durch Qualifizierungsmassnahmen und zeitliche Freiräume, welche an der intrinsischen Motivation ansetzen. Die Nutzungshäufigkeit soll jedoch auch durch externe politische Apelle oder Förderprogramme (z.B. Aufstieg durch Bildung: offene Hochschulen) erhöht werden. Da Professorinnen und Professoren in der Gestaltung ihrer Lehre frei sind und an deutschen Hochschulen zentrale Vorgaben durch deren Governancestruktur nur schwer durchsetzbar sind ist anzunehmen, dass primär intrinsische Motivationen die Einführung von E-Learning beeinflussen.

Hypothese 4: Die intrinsische Motivation hängt stärker positiv mit dem Umfang der Nutzung von virtueller Lehre zusammen als die extrinsische Motivation. 


\section{Methodik}

Als Datengrundlage dieser Arbeit dient der Datensatz einer 2017 durchgeführten quantitativen Befragung von aktiven Hochschullehrenden der Hochschule für Wirtschaft und Gesellschaft Ludwigshafen. Der Fragebogen erfasst sowohl eine Selbsteinschätzung der Kenntnisse und Fähigkeiten im Bereich virtuelle Lehre von Professorinnen und Professoren sowie Lehrbeauftragten, als auch deren Lehrerfahrung mit virtueller Lehre und ihre Einstellungen gegenüber derselben. Insgesamt beteiligten sich an der Online-Befragung 67 Personen. Dies entspricht einer Rücklaufquote von ca. $20 \%$ der Grundgesamtheit.

Die Erfassung der E-Teaching Kompetenz im Messinstrument erfolgte in Anlehnung an die oben erwähnten Kompetenzdimensionen von Horvath (2009). Da die Erfassung der E-Teaching Kompetenz nur ein kleiner Aspekt der Befragung war und noch zahlreiche andere Fragestellungen in der Befragung abzubilden waren, wurden lediglich sechs einzelne Items für die Kompetenzdimensionen ausgewählt. Die Auswahl wurde nach dem Kriterium der Relevanz für die Hochschullehrerschaft an der Hochschule für Wirtschaft und Gesellschaft Ludwigshafen auf Basis einer qualitativen Auswertung der Beratungsgespräche des Kompetenz- und Support Center E-Learning im Zeitraum 2016 bis 2017 getroffen. Es wurden nur diejenigen Items erhoben, die vom E-Learning-Team der Hochschule im Rahmen der formativen Evaluation als besonders bedeutsam für die zukünftige Ausrichtung der Beratung und Unterstützung bewertet wurden. Daher wurden die Dimensionen Kommunikationskompetenz, Teamprozesse, Planungs- und Organisationskompetenz sowie die didaktische Kompetenz vernachlässigt. Die Erhebung nimmt auf die Selbsteinschätzung der Lehrenden Bezug, da die Durchführung einer Fremdbeurteilung den Rahmen der Evaluation des E-Learning und hochschuldidaktischen Angebots gesprengt hätte. Die Validität der Selbsteinschätzung von Kompetenzen wird zwar häufig kritisch diskutiert, hat sich aber mittlerweile in vielen Studierenden- und Lehrendenbefragung (Eichhorn und Tillmann 2018) etabliert, was sich insbesondere in der Verbreitung des Instruments «BEvaKomp» zeigt (Braun und Leidner 2009; Boentert et al. 2017).

Zunächst wurden die Daten deskriptiv mit SPSS 24 ausgewertet. Dafür wurden zum Aufdecken von Abweichungen und Gruppenunterschieden bivariate Analysen durchgeführt, indem sowohl Chi-Quadrat als auch T-Tests berechnet wurden (Schnell, Hill und Esser 2011). Es wurden alle Tests zweiseitig mit der Signifikanzgrenze $p \leq 0,05$ durchgeführt. Zudem wurden multivariate Analysen durchgeführt um eine Dimensionsreduktion und die Qualität der daraus abgeleiteten Indizes zu prüfen. Die Indizes sind in die Analyse der Stärke der Zusammenhänge mit eingeflossen. Tabelle 3 liefert einen Überblick über die Verteilung der in der Analyse verwendeten Variablen. 


\begin{tabular}{|c|c|c|c|}
\hline Item & n & $\begin{array}{l}\sum \text { Ska- } \\
\text { lenwerte } \\
4+5 \text { in } \%\end{array}$ & $\bar{x}(s d)$ \\
\hline \multicolumn{4}{|c|}{$\begin{array}{l}\text { E-Teaching Kompetenzen } \\
\text { Schätzen Sie bitte Ihre eigenen Kenntnisse und Fähigkeiten in den folgenden Bereichen ein: } \\
\text { (5-er Skala: } 1 \text { = überhaupt nicht } 5=\text { in hohem Maße, . = kann ich nicht beurteilen) }\end{array}$} \\
\hline $\begin{array}{l}\text { Medientechnische Kompetenz: Lernplattformen (z.B. OLAT) } \\
\text { selbst bedienen können }\end{array}$ & 60 & $32 \%$ & $2,98(1,16)$ \\
\hline $\begin{array}{l}\text { Medientechnische Kompetenz: Kenntnisse in der Herstel- } \\
\text { lung von Lehrvideos und Audiodateien }\end{array}$ & 58 & $12 \%$ & $1,93(1,09)$ \\
\hline $\begin{array}{l}\text { Beratungskompetenz: Die Studierenden online (z.B. in } \\
\text { OLAT) beraten können }\end{array}$ & 50 & $16 \%$ & $2,28(1,16)$ \\
\hline $\begin{array}{l}\text { Mediendidaktische Kompetenz: Lernprozesse der Studie- } \\
\text { renden in der virtuellen Lehre anleiten }\end{array}$ & 51 & $8 \%$ & $2,20(1,02)$ \\
\hline $\begin{array}{l}\text { Index: Rechtliche Kompetenzen (Items: Datenschutz \& Ur- } \\
\text { heberrecht, } \alpha=0,882 \text { ) }\end{array}$ & 59 & - & $2,82(1,04)$ \\
\hline \multicolumn{4}{|c|}{$\begin{array}{l}\text { Gründe gegen die Durchführung von virtueller Lehre } \\
\text { Welche der folgenden Gründe spielen für die Entscheidung virtuelle Lehre nicht (mehr) durchzufüh- } \\
\text { ren eine Rolle? } \\
\text { (5-er Skala: } 1 \text { = überhaupt keine Rolle, } 5 \text { = eine sehr starke Rolle) }\end{array}$} \\
\hline Persönliche Präferenz für die Präsenzlehre & 32 & $78 \%$ & $4,16(1,30)$ \\
\hline Probleme bei der Umsetzung der Lehrinhalte & 29 & $52 \%$ & $3,31(1,26)$ \\
\hline Fehlende eigene technische Kompetenzen & 31 & $39 \%$ & $3,03(1,40)$ \\
\hline $\begin{array}{l}\text { Fehlende eigene Kompetenzen zur Lehrplanung virtueller } \\
\text { Angebote }\end{array}$ & 32 & $28 \%$ & $2,75(1,32)$ \\
\hline Fehlende eigene zeitliche Kapazitäten & 31 & $68 \%$ & $3,65(1,33)$ \\
\hline \multicolumn{4}{|c|}{$\begin{array}{l}\text { Gründe für die Durchführung von virtueller Lehre } \\
\text { Welche der folgenden Gründe spielen für die Entscheidung virtuelle Lehre durchzuführen eine Rolle? } \\
\text { (5-er Skala: } 1 \text { = überhaupt keine Rolle, 5 = eine sehr starke Rolle) }\end{array}$} \\
\hline Persönliches Interesse & 45 & $71 \%$ & $3,80(0,97)$ \\
\hline Politische Zielsetzung & 44 & $7 \%$ & $1,77(1,10)$ \\
\hline Zeitliche Flexibilisierung für Studierende & 45 & $71 \%$ & $3,82(1,27)$ \\
\hline Die Anwendung neuer didaktischer Möglichkeiten & 44 & $84 \%$ & $4,25(0,78)$ \\
\hline \multicolumn{4}{|l|}{$\begin{array}{l}\text { Nutzungsintensität } \\
\text { Wie häufig nutzen Sie die folgenden Funktionen von OLAT derzeit? } \\
\text { (5-er Skala. } 1 \text { = überhaupt nicht } 5=\text { sehr häufig . = kenne ich nicht) }\end{array}$} \\
\hline $\begin{array}{l}\text { Index: Nutzungsintensität (Materialordner, Videos, Aufga- } \\
\text { ben, Test, Foren, Podcasts, Blogs, Themenvergabe; } \alpha=0,725 \text { ) }\end{array}$ & 33 & - & $2,02(0,71)$ \\
\hline Materialordner & 51 & $80 \%$ & $4,22(1,33)$ \\
\hline Videos & 45 & $11 \%$ & $1,73(1,12)$ \\
\hline Aufgaben & 52 & $38 \%$ & $2,75(1,72)$ \\
\hline Tests und Selbsttests & 46 & $11 \%$ & $1,63(1,12)$ \\
\hline Foren & 47 & $11 \%$ & $1,62(1,05)$ \\
\hline Podcasts & 41 & $2 \%$ & $1,24(0,62)$ \\
\hline Blogs & 42 & $2 \%$ & $1,24(0,69)$ \\
\hline Themenvergabe & 43 & $21 \%$ & $2,09(1,49)$ \\
\hline
\end{tabular}

Tab. 3.: Übersicht über die in der Analyse verwendeten Items. 


\section{Ergebnisse}

\section{Einsatz von E-Learning und E-Teaching Kompetenzen}

Die Daten zeigen, dass E-Learning noch nicht bei allen Lehrenden der Hochschule zum Einsatz kommt. Dies trifft insbesondere auf die Lehrbeauftragten zu. In Abb. 1 wird sichtbar, dass deutlich mehr hauptamtlich Lehrende schon einmal virtuelle Lehre eingesetzt haben im Vergleich zu Lehrbeauftragten $\left(x^{2}=8,355, d f=1, p=0,005\right.$, n=61). In Bezug auf die zukünftige Nutzung ist die Gruppe an Lehrbeauftragten noch sehr unentschieden, ob sie zukünftig virtuelle Lehre einsetzen möchte, während ein Grossteil der hauptamtlich Lehrenden auch zukünftig virtuelle Lehre einsetzen möchte $\left(x^{2}=11,23, d f=2, p=0,004, n=63\right)$. Die zwei gewichtigsten Gründe gegen die Durchführung virtueller Lehre sind «persönliche Präferenz für die Präsenzlehre», gefolgt von den «fehlenden eigenen zeitlichen Kapazitäten». Fehlende Kompetenzen spielen laut den Lehrenden nur eine untergeordnete Rolle.

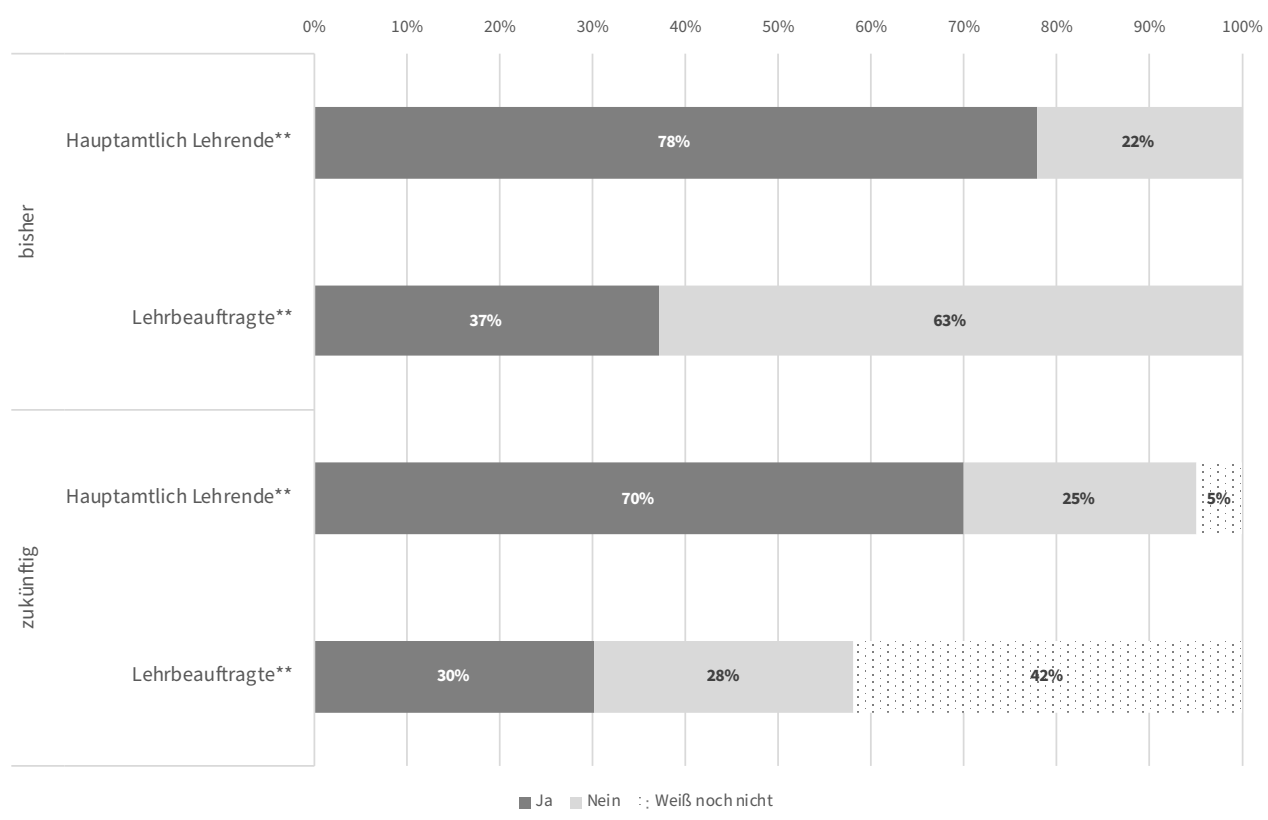

Abb. 1.: Häufigkeitsverteilung der bisherigen und zukünftigen Nutzung virtueller Lehre nach Status der Lehrenden, Chi ${ }^{2}$-Test signifikant: ${ }^{\star} p \leq 0,05,{ }^{\star \star} p \leq 0,01,{ }^{\star \star *} p \leq 0,001$.

Die Selbsteinschätzung der virtuellen E-Teaching-Kompetenzen der Lehrenden fällt insgesamt eher zurückhaltend aus (siehe Tabelle 3). Es gibt keine Kompetenzdimension, in der sich ein grösserer Teil der Lehrenden als in hohem Masse kompetent einschätzt. So liegen die Mittelwerte in der Medientechnischen Kompetenz bzgl. Lernplattformen wie auch in den rechtlichen Kompetenzen im mittleren Bereich, 
während die Mediendidaktische Kompetenz, die Beratungskompetenz und die Medientechnische Kompetenz in der Herstellung von Lehrvideos und -audiodateien insgesamt als eher niedrig eingeschätzt wird. Die relativ hohen Standardabweichungen verdeutlichen jedoch, dass die Beurteilung nicht einheitlich ausfällt, sodass es auch vereinzelt Lehrende gibt, die ihre Kompetenzen in diesen Bereichen höher einschätzen. Die Präferenz für die Präsenzlehre spiegelt sich auch sehr deutlich in dem Nutzungsverhalten des Lernmanagementsystems (LMS) OLAT wieder. Es zeigt sich deutlich, dass dieses LMS an der Hochschule u.a. zur Ergänzung der Präsenzlehre genutzt wird, indem insbesondere die Materialordner, in denen beispielsweise Skripte oder Texte eingestellt werden können, (sehr) häufig genutzt werden. Andere Funktionen, die sich gut für die Begleitung von Lernprozessen in einer Online-Umgebung eignen (wie Aufgaben, Blogs, Themenvergabe oder Tests), kommen aktuell nur bei sehr wenigen Lehrenden zur Anwendung. Hypothese 1 wird durch die Daten bestätigt.

\section{Nutzungsintensität und Motivation}

Die Analyse von Korrelationen zeigt, dass die Nutzungsintensität von OLAT (Index Nutzungsintensität) nur mit zwei Dimensionen der E-Teaching-Kompetenzeinschätzung zusammenhängt, nämlich der Medientechnischen Kompetenz der Bedienung von Lernplattformen $(r=0,578, p=0,000, n=33)$ und der Mediendidaktischen Kompetenz $(r=0,556, p=0,002, n=28)$. Bei den anderen erhobenen Kompetenzen lässt sich kein Zusammengang mit der Nutzungsintensität feststellen. Hypothese 2 kann daher nicht für alle abgefragten E-Teaching-Kompetenzen bestätigt werden.

Da die Nutzungsintensität eng mit dem Status der Lehrenden zusammenhängt, ist eine Überprüfung des Zusammenhangs von E-Teaching-Kompetenz und Status sinnvoll. Es wird deutlich, dass hauptamtlich Lehrende sich deutlich kompetenter einschätzen als Lehrbeauftragte - ausser bei der rechtlichen Kompetenz (siehe Abb.2). Die Mittelwerte unterscheiden sich insbesondere bei der Medientechnischen Kompetenz bzgl. der Lernplattformen und der virtuellen Lernbegleitung. Etwas weniger ausgeprägt sind die Unterschiede in der Beratung und der Produktion von Lehrvideos oder-audios). Hypothese 3 lässt sich - mit Ausnahme der rechtlichen Kompetenzen - bestätigen. 


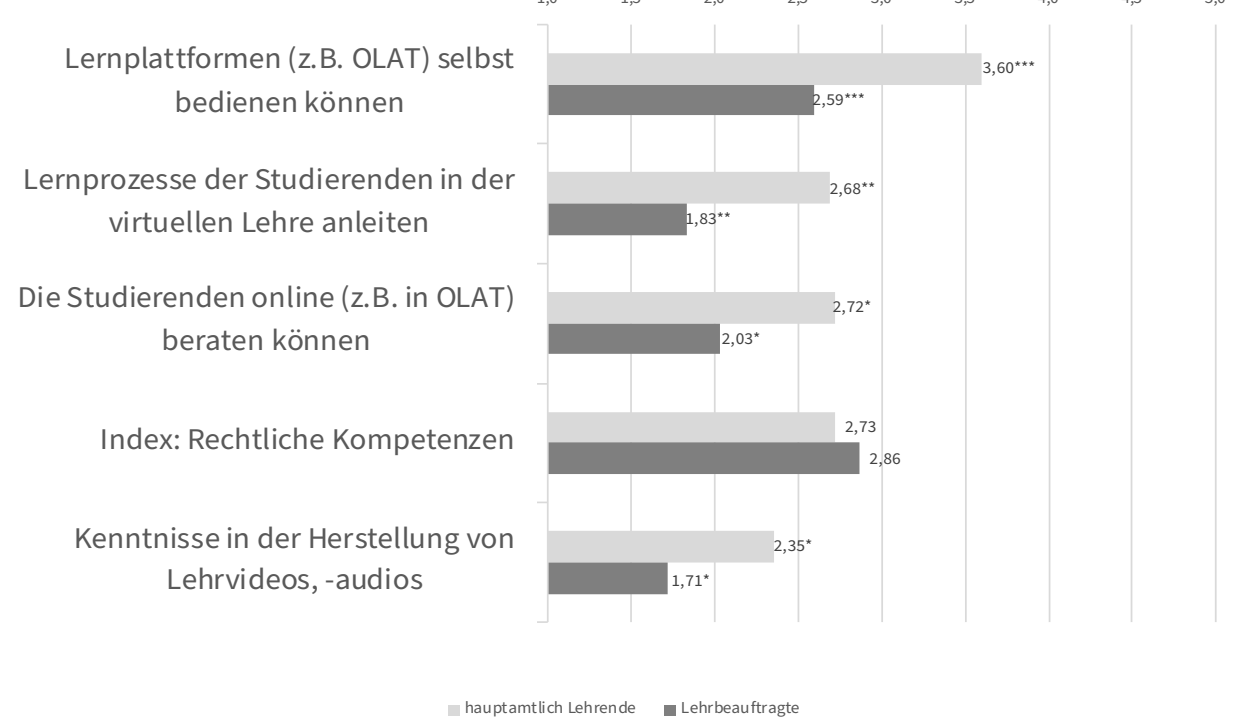

Abb. 2.: Mittelwerte der E-Teaching Kompetenzen nach Status der Lehrenden, T-Test signifikant: ${ }^{*} \mathrm{p} \leq 0,05,{ }^{* *} \mathrm{p} \leq 0,01,{ }^{\star * *} \mathrm{p} \leq 0,001$.

Betrachtet man die Gründe, die laut den Lehrenden für die Durchführung von E-Learning sprechen, wird deutlich, dass die Anwendung neuer didaktischer Möglichkeiten, das persönliche Interesse und die zeitliche Flexibilisierung die wichtigsten Gründe für E-Learning sind. Dennoch hängt lediglich die intrinsische Motivation (das eigene Interesse) signifikant mit der Nutzungshäufigkeit zusammen ( $r=0,438, p=0,028, n=$ 25). Es zeigt sich ausserdem, dass die intrinsische Motivation deutlich höher ist als die extrinsische Motivation, wobei letztere nicht mit der Nutzungsintensität zusammenhängt ( $r=-0,249, p=0,241, n=24)$. Hypothese 4 kann daher bestätigt werden.

\section{Diskussion}

Die Ergebnisse zeigen, dass die Hochschullehrenden besonders diejenigen ETeaching Kompetenzen als hoch einschätzen, die mit angereicherter Lehre zusammenhängen und die sie häufig nutzen. Die Verlagerung eines grösseren Anteils einer Lehrveranstaltung in den digitalen Raum bedeutet aber viel mehr, zum Beispiel eine dem Onlinemedium und den Lernereigenschaften angepasste Gestaltung der Lernmaterialien, eine lernprozessförderliche Gestaltung des Lehr-Lernszenarios und eine gute (online-)Beratung und Betreuung der Studierenden. Da die Lehrenden die hierfür benötigten Kompetenzen als ausbaufähig einschätzen, sollte ein Fokus auf Weiterentwicklung dieser Kompetenzen liegen. Eine Möglichkeit hierfür ist es, den Hochschullehrenden Erfahrungsräume für das Anwenden und Umsetzen von E-Learning zu bieten. Das Lernen von und mit Peers - entweder informell oder auch angeleitet in Workshops - könnte dafür eine gute Möglichkeit sein (Bremer 2003). 
Doch wie kann die Politik und können Hochschulen über Qualifizierungsangebote hinaus die Entwicklung und Umsetzung von E-Learning wirksam unterstützen? Schon vor über 15 Jahren hat Bremer (2002) darauf hingewiesen, dass «bestehende Widerstände bezüglich dieser neuen Rolle [...] nur im Lauf der Zeit und mit einem erfolgreichen Change Management überwunden werden» können (Bremer 2002, 126). Die Ergebnisse bestätigen diese Einschätzung und zeigen, dass die intrinsische Motivation das ausschlaggebende Argument für den Einsatz von E-Learning darstellt. Dies kann ein generelles persönliches Interesse sein, aber auch lehrbezogen die Möglichkeit neue didaktische Konzepte und Tools zu nutzen. Hochschulen sollten darüber hinaus angemessene Rahmenbedingungen schaffen und somit den Lehrenden genügend Zeit und eine gute technisch-didaktische Unterstützung bereitstellen. Die Anrechnung des Erstellungs- und Durchführungsaufwandes auf das Lehrdeputat ist dabei eine gute Option, um den hohen Anfangsaufwand abzufedern (Umsetzung siehe Lungershausen, Emunds und Buß 2016). Für die politischen Entscheidungsträgerinnen und Entscheidungsträger hingegen gilt es, diese guten Rahmenbedingungen an den Hochschulen zu unterstützen. Politische Appelle oder Zielvereinbarungen mit den Hochschulen sind nur für eine marginale Anzahl an Hochschullehrenden ein Grund E-Learning einzuführen. Projekte zur Einführung von Blended-Learning müssen daher an der intrinsischen Motivation der Lehrenden ansetzen und eine umfassende Unterstützung bei der Konzeption und Umsetzung von Kursen anbieten und damit die Eigeninitiative der Lehrenden möglichst sinnvoll unterstützen.

Schliesslich bieten die Lehrbeauftragten ein hohes Potential, um E-Learning auszubauen. Denn diese Zielgruppe ist häufig unentschlossen, ob sie E-Learning anwenden möchte. Da die E-Teaching Kompetenzen der Lehrbeauftragten im Durchschnitt recht gering sind und ihre Hauptaufgabe nicht in der Hochschullehre liegt, ist dieses Potential allerdings schwer zu heben. Eine Fokussierung auf langjährige Lehrbeauftragte mit einem höheren SWS-Umfang scheint angesichts der Kosten für die Qualifizierung und Beratung derselben und des Erstellungsaufwands für die E-Learning Kurse angebracht.

Bei der Interpretation der Ergebnisse muss einschränkend beachtet werden, dass die Stichprobe recht klein ist und die Daten nur an einer Hochschule erhoben wurden.

\section{Literatur}

Arnold, Patricia, Lars Kilian, Anne Thillosen, und Gerhard Zimmer. 2018. Handbuch für E-Learning: Lehren und Lernen mit digitalen Medien. 5. Aufl. Bielefeld: Bertelsmann Verlag.

Bloom, B. S. und M. D. Engelhart. 1976. Beltz-Studienbuch: Vol. 35. Taxonomie von Lernzielen im kognitiven Bereich. 5. Aufl. Weinheim: Beltz. 
Boentert, Annika, Therese Kirsch, Sven Niehues, und Jutta Rach. 2017. «Kompetenzorientierte Evaluation - entzaubert? Probleme und Lösungsansätze.» Handbuch für Qualität in Studium und Lehre (59): 1-21.

Braun, Edith, und Bernhard Leidner. 2009. «Academic Course Evaluation.» European Psychologist 14 (4): 197-306. https://doi.org/10.1027/1016-9040.14.4.297.

Bremer, Claudia. 2002. "Qualifizierung Zum EProf? Medienkompetenz Und Qualifizierungsstrategien Für Hochschullehrende.» In Campus 2002: Die virtuelle Hochschule in der Konsolidierungsphase, herausgegeben von Gudrun Bachmann, 123-36. Medien in der Wissenschaft Bd. 18. Münster: Waxmann.

Bremer, Claudia. 2003. «Hochschullehre und Neue Medien: Medienkompetenz und Qualifizierungsstrategien für Hochschullehrende.» In Hochschuldidaktische Aus- und Weiterbildung, herausgegeben von Ulrich Welbers, 323-45. Gütersloh: Bertelsmann.

Busse, Brigitta. 2017. «E-Learning an Hochschulen: Stand der Entwicklung unter didaktischer Perspektive.» Hefte zur Bildungs- und Hochschulforschung 90.

Carstensen, Doris. 2009. «Wandel und E-Learning in Hochschulen - überraschende Transformationsmuster.»In E-Learning: Eine Zwischenbilanz: Kritischer Rückblick als Basis eines Aufbruchs, herausgegeben von Ullrich Dittler, J. Krameritsch, N. Nistor, C. Schwarz und A. Thillosen, 249-61. Münster/New York/München/Berlin: Waxmann.

Dengel, Andreas. 2018. «Digitale Bildung: ein interdisziplinäres Verständnis zwischen Medienpädagogik und Informatik.» MedienPädagogik 33: 11-29. https://doi.org/10.21240/ mpaed/33/2018.10.30.X.

Eichhorn, Michael, Ralph Müller, und Alexander Tillmann. 2017. «Entwicklung eines Kompetenzrasters zur Erfassung der «Digitalen Kompetenz» von Hochschullehrenden.» 25. Jahrestagung der Gesellschaft für Medien in der Wissenschaft, Chemnitz, 2017.

Eichhorn, Michael und Alexander Tillmann, Hrsg. 2018. Digitale Kompetenzen von Hochschullehrenden messen: Validierungsstudie eines Kompetenzrasters.

Ferrari, Anusca. 2012. "Digital Competence in Practice: An Analysis of Frameworks.» http:// jiscdesignstudio.pbworks.com/w/file/fetch/55823162/FinalCSReport_PDFPARAWEB.pdf.

Hermida, Martin, Michael Hielscher, und Dominik Petko. 2017. «Medienkompetenz messen: Die Entwicklung des Medienprofis-Tests in der Schweiz.» MedienPädagogik, 38-60. https://doi.org/10.21240/mpaed/00/2017.06.02.X.

Horvath, Eva. 2009. «Was macht E-Learning erfolgreich? Erfassung und Förderung von E-Lehrkompetenz für die Hochschullehre.».

Hugger, Kai-Uwe. 2004. «Neue Medien und Hochschullehrer. Zu Hindernisgründen und Widerständen.» In Medienkompetenz für die Hochschullehre, herausgegeben von Katja Bett, Joachim Wedekind und Peter Zenkel, 269-274. Münster: Waxmann.

Hugger, Kai-Uwe, und R. Vollbrecht. 2001. «Neue Medien - neue Lehrerausbildung? Ergebnisse einer Expertenbefragung." In Lehrerausbildung und neue Medien. Erfahrungen und Ergebnisse eins Hochschulnetzwerks, herausgegeben von U. Bentlage und I. Hamm, 29-44. Gütersloh: Bertelsmann.

Kerres, Michael, und Barbara Getto. 2017. «Vom E-Learning Projekt zur nachhaltigen Hochschulentwicklung; Strategisches Alignment im Kernprozess «Studium und Lehre».»In Hochschulwege 2015: Wie verändern Projekte die Hochschulen? herausgegeben von Andreas Mai. 1. Auflage, 147-57. Hamburg: tredition. 
Kleimann, Bernd, und Klaus Wannemacher. 2004. E-Learning an deutschen Hochschulen: Von der Projektentwicklung zur nachhaltigen Implementierung. Hochschulplanung 165. Hannover: HIS. http://www.his.de/pdf/pub_hp/hp165.pdf.

Kohl, Kerstin E. 2004. «Entwicklung einer Strategie für die didaktische Begleitung von E-Learning-Vorgaben zur Virtualisierung der Hochschullehre am Beispiel des Forschungsprojektes ITO.» Dissertation, Pädagogische Hochschule Ludwigsburg.

Kreidl, Christian. 2011. Akzeptanz und Nutzung von E-Learning-Elementen an Hochschulen: Gründe für die Einführung und Kriterien der Anwendung von E-Learning. Münster/New York/ München/Berlin: Waxmann.

Kultusministerkonferenz. 2016. «Bildung in der digitalen Welt.» https://www.kmk.org/fileadmin/Dateien/pdf/PresseUndAktuelles/2017/Strategie_neu_2017_datum_1.pdf.

Lungershausen, Uta, Georg Emunds, und Imke Buß. 2016. «Anrechnung virtueller Lehre auf das Lehrdeputat.» Die neue Hochschule (4): 102-5.

Redecker, Christine. 2017. «European Framework for the Digital Competence of Educatiors: DigCompEdu.» https://ec.europa.eu/jrc/en/digcompedu.

Satow, Lars. 2002. «Unterrichtsklima und Selbstwirksamkeitsdynamik.» Zeitschrift für Pädagogik 44: 174-91.

Sauter, Annette, Werner Sauter, und Harald Bender. 2003. Blended-Learning. Effiziente Integration von E-Learning und Präsenztraining. München: Luchterhand.

Schmid, Ulrich, Lutz Goertz, Sabine Radomski, Sabrina Thom, und Julia Behrens. 2017. «Monitor Digitale Bildung: Die Hochschulen im digitalen Zeitalter.»

Schnell, Rainer, Paul B. Hill, und Elke Esser. 2011. Methoden der empirischen Sozialforschung. 9., aktualisierte Aufl. München: Oldenbourg.

Schöler, Stefanie. 2016. «Akademische Lehrkompetenz: Modellierung, Entwicklung und Messung mit Mixed-Methods.»

Tulodziecki, Gerhard. 2012. «Medienpädagogische Kompetenz und Standards in der Lehrerbildung.» In Jahrbuch Medienpädagogik 9, herausgegeben von Renate Schulz-Zander, Birgit Eickelmann, Heinz Moser, Horst Niesyto und Petra Grell, 271-97. Wiesbaden: VS Verlag für Sozialwissenschaften.

United Nations Educational. 2018. «UNESCO ICT Competency Framework for Teachers.» https://unesdoc.unesco.org/ark:/48223/pf0000265721.

Vonken, Matthias. 2015. Handlung und Kompetenz: Theoretische Perspektiven für die Erwachsenen- und Berufspädagogik. [S.I.]: VS Verlag für Sozialwissenschaften.

\section{Förderhinweis}

Das dieser Veröffentlichung zugrundeliegende Vorhaben wurde mit Mitteln des Bundesministeriums für Bildung und Forschung unter dem Förderkennzeichen $160 H 21050$ gefördert. Die Verantwortung für den Inhalt dieser Veröffentlichung liegt bei den Autoren. 